\title{
Gradhiva
}

\section{Frédéric Léotar, La Steppe musicienne. Analyse et modélisation du patrimoine musical turcique}

Paris, Vrin, coll. « MusicologieS », 2014

\section{Laurent Legrain}

\section{(2) OpenEdition \\ Journals}

Édition électronique

URL : http://journals.openedition.org/gradhiva/3212

DOI : $10.4000 /$ gradhiva.3212

ISSN : 1760-849X

\section{Éditeur}

Musée du quai Branly Jacques Chirac

Édition imprimée

Date de publication : 25 mai 2016

Pagination : 234-235

ISBN : 978-2-35744-093-7

ISSN : 0764-8928

\section{Référence électronique}

Laurent Legrain, « Frédéric Léotar, La Steppe musicienne. Analyse et modélisation du patrimoine musical turcique », Gradhiva [En ligne], 23 | 2016, mis en ligne le 25 mai 2016, consulté le 24 septembre 2020. URL : http://journals.openedition.org/gradhiva/3212 ; DOI : https://doi.org/10.4000/gradhiva.3212

Ce document a été généré automatiquement le 24 septembre 2020.

(c) musée du quai Branly 


\section{Frédéric Léotar, La Steppe} musicienne. Analyse et modélisation du patrimoine musical turcique

Paris, Vrin, coll. « MusicologieS », 2014

\section{Laurent Legrain}

\section{RÉFÉRENCE}

Frédéric Léotar, La Steppe musicienne. Analyse et modélisation du patrimoine musical turcique. Paris, Vrin, coll. « MusicologieS », 2014, 300 p. 


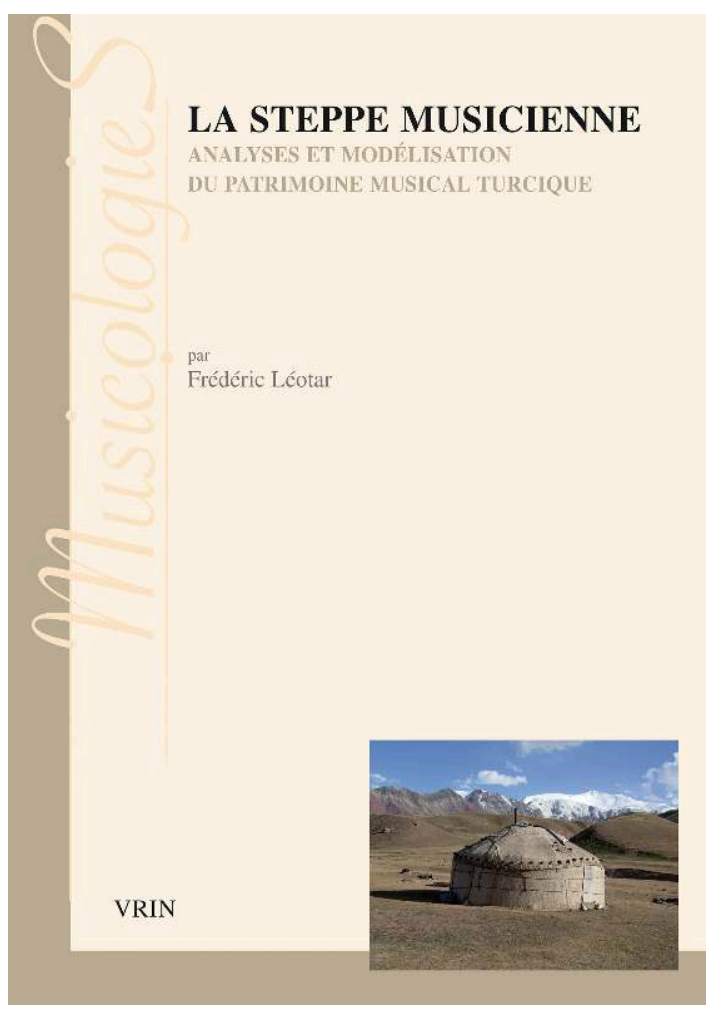

1 La Steppe musicienne: le titre de l'ouvrage de Frédéric Léotar est un peu trompeur. En effet, ce n'est pas tant la steppe qui est musicienne que les populations turciques qui la peuplent, de la Sibérie méridionale à l'ouest du Kazakhstan. Dans le premier chapitre, pourtant, le vent de la steppe s'engouffre dans les rues de Kyzyl, capitale touvaine, faisant résonner le luth d'un chanteur du nom d'Ondar Mongun-ool. Épiphanie de la musique et moment de "ravissement contagieux », souligne l'auteur (p. 28). Au fil des pages de ce chapitre d'ouverture, «La place de la musique dans la vie quotidienne et rituelle ", on entendra encore s'écouler l'eau d'une rivière et les chanteurs imiter le son de leur environnement direct, dont le ronronnement d'une écrémeuse (p. 30). L'attention de l'auteur se porte ensuite sur les mélodies huchées. Puis la steppe se tait alors que la présentation des techniques vocales curatives, des adresses aux esprits et des berceuses clôture calmement ce chapitre. On suppose que les pratiques présentées forment une portion congrue de la musique qui anime cette aire géographique immense dont l'auteur entend traiter.

2 Au-delà de ce chapitre introductif, c'est plutôt ce que les chanteurs ont dans la tête qui intéresse au premier chef Léotar. Et ce qu'ils ont dans la tête est un modèle mélodique de référence qu'ils actualisent au gré de leurs chants en s'appuyant sur les trois formes de mémoire - à court terme, à moyen terme, à long terme - que la cognition musicale explore depuis des années. Ce modèle mélodique de référence trouve sa plus simple expression chez les Touvas (chapitre III) et se complexifie à mesure que l'on avance vers l'ouest en traversant le Kirghizstan (chapitre IV), l'Ouzbékistan (chapitre v) et la république autonome du Karakalpakstan (chapitre VI), pour achever le périple musical proposé par l'auteur au Kazakhstan (chapitre VII). En chemin, l'ethnomusicologue nous a présenté soixante-huit pièces musicales puisant dans les répertoires variés - mélodies huchées, berceuses, lamentations, chants de berger, mélodies instrumentales, etc. - des populations qu'il rencontre sans ménager sa peine. Le voyage s'achève parfois sur le dos d'un âne ou à pied car les chanteurs que Léotar rencontre ont, malgré les 
vicissitudes de décennies d'une histoire socialiste commune, conservé un mode de vie pastoral ou agropastoral. Le lecteur peut écouter l'ensemble du corpus enregistré entre 1996 et 2011 en accédant au site internet associé à l'ouvrage. Les enregistrements sont beaux et poignants, les voix superbement captées par les micros de Léotar.

L'analyse et la modélisation du patrimoine musical turcique sont à la fois le sous-titre et le vrai sujet de l'ouvrage. L'une et l'autre occupent l'auteur de la page 61 à la page 206 , laissant au très beau dernier chapitre "Structures, musique, contexte » le soin d'orchestrer la rencontre entre " esprit de géométrie » et " esprit de finesse », comme disait Philippe Descola (Descola 1986). L'hypothèse selon laquelle il existerait un modèle sous-jacent ou, pour le dire autrement, que les mélodies enregistrées par l'auteur partagent les mêmes caractéristiques structurelles constitue le moteur de la recherche. Il s'agit donc de découvrir sous le buissonnement des variations des formules mélodico-rythmiques plus stables. Cette perspective de recherche inspirée de l'analyse paradigmatique de Nicolas Ruwet vise à mettre en lumière les procédés de "répétition-transformation " à l'œuvre à l'intérieur d'une pièce musicale mais aussi « d'une pièce à l'autre, pour un(e) même interprète, entre les musicien(ne)s d'un même village, d'une même région, et finalement entre toutes les pièces considérées [...]» (p. 65). L'auteur décrit ce mouvement analytique comme "une mise en paradigme généralisée » qui le mène à penser que «les agro-pasteurs fonctionnent à partir d'un stock dans lequel tous vont puiser » (ibid.). L'affinité de la méthode avec la grammaire générative de Noam Chomsky et l'analyse structurale de Claude Lévi-Strauss est clairement revendiquée et, en effet, l'écho de la voix de l'anthropologue français s'impose à l'esprit du lecteur car ici aussi il s'agit bien de "distinguer un donné purement phénoménologique [...] d'une infrastructure plus simple que lui et à laquelle il doit toute sa réalité » (Lévi-Strauss 1989 [1950] : XXXV). Du côté de l'analyse musicale, la filiation remonte à Jean Molino, Jean-Jacques Nattiez, Ruwet et Simha Arom en passant par Leonard B. Meyer et son concept de notes structurelles que Léotar adapte à l'analyse de son propre corpus. Les notes structurelles (les seules qui comptent vraiment pour la mise en paradigme) ne sont pas définies à partir d'un critère métrique (leur positionnement sur un temps fort) mais de leur longueur et de leur réitération au sein de formules mélodico-rythmiques cycliques. Ces considérations sur la méthode sont exposées dans un chapitre intitulé «Questions de méthodologie » dont la densité donne le ton des chapitres suivants. L'auteur va droit au but, sans fioriture aucune, mais fournit à son lecteur l'ensemble du matériel qui doit lui permettre de suivre les développements analytiques et, éventuellement, de repérer là où il lui semble que l'objet musical est un peu trop trituré. Ce fut le cas lors de ma lecture pour le traitement réservé aux notes ornementales échappées, ces notes détachées de leur cellule théorique et acceptées dans d'autres cellules comme " locataires temporaires » (p. 92 sqq.).

4 L'ouvrage est construit comme une démonstration. L'argumentaire est solide et intense, et a le côté rassurant d'une science cumulative qui va de l'avant et découvre le modèle qu'elle avait postulé et pour lequel elle avait conçu ses outils d'analyse. Ainsi mis en paradigme, l'ensemble du patrimoine musical turcique apparaît reposer sur les combinaisons de cinq cellules de base symbolisées par les lettres A, B, C, D et E. Le modèle le plus simple, $\mathrm{ABCBA}$, montre à la fois l'ampleur du stock de cellules auquel puise le chanteur touvain et met à plat l'ordonnancement temporel de celles-ci. Chaque chant n'actualise pas le modèle dans son entièreté, chaque ligne mélodique non plus. Le plus petit dénominateur est formé par la structure $\mathrm{CB}$, qui constitue le seuil en dessous 
duquel le chanteur touva ne peut plus construire de mélodie (p. 92). Le modèle ABCBA est une épure qui "n'a pas de réalité tangible» (p. 73) mais révèle un modèle de référence où les trois systèmes de mémoire viennent s'alimenter. La mémoire à long terme puise dans le stock de toutes les musiques déjà entendues, la mémoire à moyen terme emprunte aux éléments musicaux de pièces jouées dans la même circonstance d'exécution et la mémoire à court terme agit à son tour lorsque le chanteur puise dans le stock des cellules qu'il vient d'utiliser dans la même pièce (p. 89). À la différence d'Arom, qui modélise par soustraction, Léotar modélise par soustraction (il procède à une épure du motif musical) et superposition théorique des cellules. Prenons à nouveau l'exemple touva puisqu'il forme la pierre de touche de tout l'édifice. L'auteur analyse treize mélodies qui n'ont pas toutes la même structure. Le modèle final est construit par superposition de ces structures selon le tableau suivant :

\begin{tabular}{|l|l|l|l|l|l|}
\hline Mélodies 1 et 2 & A & B & C & B & \\
\hline Mélodies 3 à 8 & & B & C & B & \\
\hline Mélodies 9 à 11 & & & C & B & \\
\hline Mélodies 12 et 13 & & B & C & B & A \\
\hline Modèle & A & B & C & B & A \\
\hline
\end{tabular}

5 Le modèle à l'œuvre dans les répertoires des chants touvas, représenté par la formule $A B C B A$, connaît une série de développements structurels en arche (ici symbolisés par des crochets) pour devenir ABC[DC]BA chez les Kirghizes, les Ouzbeks et les Kazakhs, et ABC [DEDC]BA chez les Karakalpaks.

6 Mon compte rendu serait bien incomplet si je ne vous parlais pas d'Asèle, charmant nourrisson de 7 mois, difficile à endormir parce que, selon les dires de sa grand-mère la très rythmique Mme A' welbekova -, elle écoute moins attentivement que ses autres petits-enfants les berceuses qu'elle lui chante. La vidéo de l'endormissement du bébé emmailloté est riche d'enseignements, tout comme l'est l'analyse de Léotar qui va ouvrir le modèle décrit jusque-là à des influences diverses, y compris extra-musicales. Dans ce chapitre, le lecteur sentira battre le pouls des Karakalpaks et de toutes ces populations turciques qui peuplent la steppe. Au risque de perdre mon propre lecteur, je vais me livrer à une énumération de l'ensemble des influences auxquelles le modèle est maintenant soumis : la métrique du poème, les interjections propitiatoires, les souvenirs de Mme A' welbekova qui la poussent à emprunter les vers de son poème à divers contextes de la vie quotidienne et aux images de virilité masculine (peu importe qu'Asèle soit une fille, la berceuse englobe la femme dans le rêve de l'homme, comme l'écrivait Roberte Hamayon pour les vœux et augures mongols [1979: 122]), les réactions d'Asèle, l'ennui et la fatigue qui commencent à gagner la grand-mère (elle observe scrupuleusement les prescriptions du jeûne en cette période de ramadan), son évaluation du stade d'endormissement du bébé. Léotar met en relation l'instabilité du " thème 3 » de la berceuse chantée et celle de l'état d'Asèle, entre éveil et sommeil. Donald Winnicott aurait applaudi des deux mains. Dans ce très beau chapitre, l'écologie intersubjective qui représente à mon sens le seul milieu viable pour la musique est pleinement prise en compte et donne une forte densité analytique au modèle dégagé le 
long des chapitres précédents. Lorsque l'auteur rencontre à nouveau cette famille en 2010, Asèle est âgée de 3 ans et vient encore vers sa grand-mère avec son oreiller en faisant avec sa langue «le mouvement caractéristique des berceuses» (p. 218). Un autre mouvement caractéristique des berceuses, que l'on peut considérer comme de véritables "techniques du corps ", est constitué par le balancement du berceau. Peutêtre Léotar pourrait-il prendre en considération cette rythmique sous-jacente à l'exécution de la ligne mélodique.

Quoi qu'il en soit, ce chapitre de clôture est un véritable régal et l'on se surprend à attendre le tome II de La Steppe musicienne qui refera le même parcours, en voiture, à dos d'âne ou à pied, et nous parlera avec verve des influences auxquelles chaque chant du corpus est soumis, auxquelles chaque modèle s'ouvre avec bonheur. Le travail qu'a réalisé Léotar est immense, celui qui reste à réaliser est sans limite comme l'est, dans l'esprit des poètes mongols, la steppe elle-même.

\section{BIBLIOGRAPHIE}

\section{Descola, Philippe}

1986 La Nature domestique. Symbolisme et praxis dans l'écologie des Achuar. Paris, Éditions de la Maison des sciences de l'homme.

\section{Hamayon, Roberte}

1979 « Le pouvoir des hommes passe par la "langue des femmes". Variation mongole sur le duo de la légitimité et de l'aptitude », L’Homme 9(3-4) : 109-139.

Lévi-Strauss, claude

1989 [1950] «Introduction à l'œuvre de Marcel Mauss », in Sociologie et anthropologie. Paris, Presses Universitaires de France ("Quadrige ») : IX-LII.

\section{AUTEURS}

\section{LAURENT LEGRAIN}

Laurent.Legrain@ulb.ac.be 